\title{
Wigner crystal induced by dipole-dipole interaction in one-dimensional optical lattices
}

\author{
Zhihao $\mathrm{Xu}^{1, \text { * }}$ and $\mathrm{Shu} \mathrm{Chen}^{1, \oplus}$ \\ ${ }^{1}$ Beijing National Laboratory for Condensed Matter Physics, \\ Institute of Physics, Chinese Academy of Sciences, Beijing 100190, China
}

(Dated: September 17, 2018)

\begin{abstract}
We demonstrate that the static structure factor, momentum distribution and density distribution provide clear signatures of the emergence of Wigner crystal for the fermionic dipolar gas with strongly repulsive dipole-dipole interactions trapped in one-dimensional optical lattices. Our numerical evidences are based on the exact diagonalization of the microscopic effective lattice Hamiltonian of few particles interacting with long-range interactions. As a comparison, we also study the system with only nearest-neighbor interactions, which displays quite different behaviors from the dipolar system in the regime of strong repulsion.
\end{abstract}

PACS numbers: 03.75.Hh,05.30.Fk,71.10.Fd

\section{INTRODUCTION}

Ultracold dipolar atomic and molecular gases with long-range interactions have become a very active research field of cold atom physics in past years. Dipolar interactions with long-range anisotropic character have been observed in Chromium atoms [1, 2]. More recently, the development of stimulated Raman adiabatic passage technique has succeeded in creating a nearly degenerate gas of heteronuclear polar molecule gases which have much greater dipole momentum [3-5] and thus are promising candidates with very strong dipolar interactions. The experimental progress has stimulated theoretical studies of dipolar gases with long-range anisotropic interactions. Loading ultracold polar molecules into optical lattices also provides a fascinating platform for studying quantum many-body systems with long range interactions.

In comparison with the short-range and isotropic interactions, dipole-dipole interactions (DDIs) may induce many new effects and phases in quantum gases due to their long-range and anisotropic character. There have been many theoretical works on, for example, the polarized dipolar Bose-Einstein condensations (BECs) [68], spinor-dipolar BECs 9-11], supersolid [12 16], s-wave scattering resonances 17], quantum Hall effects [18] and one-dimensional (1D) dipolar systems 19 23]. In the presence of long range interaction, an interesting issue is the emergence of the Wigner crystal phase. A Wigner crystal is a crystalline phase of electrons first predicted by Wigner [24]. As the interacting potential energy dominates the kinetic energy at low densities, the particles tend to form a regular crystal due to the strong longrange interaction. Wigner crystal in one dimension with long range interactions has been theoretically studied by using different methods, including bosonization [25 28], quantum Monte Carlo (QMC) [20, 21, 29, 30] and exact

\footnotetext{
*Electronic address: xuzhihao85@gmail.com
}

†Electronic address: schen@aphy.iphy.ac.cn diagonaliztion [22]. In the seminal work of Schulz [25], it has been clarified that the Coulomb long-range repulsion enhances the $4 k_{F}$ charge density correlations and drives the system to the Wigner crystal phase.

In this paper, we study the fermionic dipolar gas with strong dipole-dipole interactions trapped in 1D deep optical lattices, which can be effectively described by a Fermi lattice model with long-range interactions falling off as $1 / x^{3}$. For 1D fermionic models, the case of interactions falling off as $1 / x^{\alpha}$ has been studied in the scheme of bosonization [26, 27]. Treating long-range forward scattering as a perturbation, they find that for $\alpha>1$ the long-range forward scattering is an irrelevant perturbation. As the above conclusion is obtained based on the perturbation analysis of the low-energy effective Luttinger liquid theory with linear dispersion, it does not exclude the existence of Wigner crystal phase in the presence of strong dipole-dipole interactions. In this work, we shall study the ground state properties of the Fermi lattice model with long-range interactions by means of the exact diagonalization method. To see clearly the effect of the long-range interaction, we carefully compare the long-range interacting dipolar systems against the Fermi systems with short-range interactions. Our results display significantly different behaviors for the Fermi systems with long-range and short-range interactions in the strongly interacting regime. By observing the differences of static structure factor, momentum distribution and density distribution, we give clear evidences for the emergence of Wigner crystal for dipolar fermions with strong dipole-dipole interactions.

The paper is organized as follows. In section II, we derive the effective lattice model for the fermionic dipolar gas in a 1D optical lattice. In section III, we present our results for both the dipolar Fermi system with long range interaction and system with only nearest-neighbor (NN) interaction by using the exact diagonalization method and compare their different behaviors with increasing the interaction strength. A summary is given in the last section. 


\section{MODEL HAMILTONIAN}

Let us consider a system of electric or magnetic dipoles confined in a quasi-1D optical lattice along the $x$ direction. The interaction between dipoles aligned in the $x-z$ plane by a homogeneous filed can be simplified to

$$
V_{d d}(\vec{r})=C_{d d} \frac{1-3 \cos ^{2} \theta_{r d}}{r^{3}}
$$

where $\cos \theta_{r d}=\vec{r} \cdot \vec{d} /(r d)$ and $C_{d d}$ measures the strength of the DDI. The strength of the DDI is given by $C_{d d}=d^{2} /\left(4 \pi \varepsilon_{0}\right)$ for two electric dipoles, and by $C_{d d}=$ $\mu_{0} d^{2} /(4 \pi)$ for the magnetic ones, with $\varepsilon_{0}$ and $\mu_{0}$ being the electric constant and magnetic constant, respectively. In the single-mode approximation with only the transversal ground state considered, we can integrate over transversal directions and get the effective $1 \mathrm{D}$ DDI given by $V_{d d}=U_{d d} \widetilde{V}_{d d}\left(|x| / l_{\perp}\right)$, with $U_{d d}=$ $-C_{d d}[1+3 \cos (2 \theta)] /\left(8 l_{\perp}^{3}\right), \quad \widetilde{V}_{d d}(u)=-2 u+\sqrt{2 \pi}(1+$ $\left.u^{2}\right) \exp \left(u^{2} / 2\right) \operatorname{erfc}(u / \sqrt{2})$ and $l_{\perp}=\sqrt{\hbar /\left(m \omega_{\perp}\right)}$ [19, 22].

The Hamiltonian for dipolar fermions in optical lattices is given by

$$
\begin{aligned}
H= & \int \mathrm{d} x \psi^{\dagger}(x)\left[-\frac{\hbar^{2}}{2 m} \frac{\partial^{2}}{\partial x^{2}}+V_{0}(x)\right] \psi(x) \\
& +\frac{1}{2} \iint \mathrm{d} x \mathrm{~d} x^{\prime} \psi^{\dagger}(x) \psi^{\dagger}\left(x^{\prime}\right) V_{d d}\left(x-x^{\prime}\right) \psi\left(x^{\prime}\right) \psi(x)
\end{aligned}
$$

where $m$ is the mass of the dipolar fermions, $\psi(x)$ is a fermionic field operator for the dipolar fermion, and $V_{0}(x)$ is the optical lattice field given by $V_{0}(x)=$ $V_{0} \sin ^{2}(k x)$ with the wavevectors $k=2 \pi / \lambda$ and the wavelength of the laser light $\lambda$. Considering the deep optical lattice with particles trapped in the lowest vibrational state $\omega(x)=\exp \left(-x^{2} /\left(2 l_{x}^{2}\right)\right) /\left(\pi^{1 / 4} \sqrt{l_{x}}\right)$ with $l_{x}=\sqrt{\hbar /\left(m \omega_{x}\right)}$ and $\hbar \omega_{x}=2 \sqrt{E_{R} V_{0}}$, we use the Wannier basis to expand the field operator $\psi(x)=\sum_{i} c_{i} \omega\left(x-x_{i}\right)$. Similar to the case of Hubbard model [31], we can get the effective lattice Hamiltonian for the polar fermionic system

$$
H=-J \sum_{i}\left(c_{i}^{\dagger} c_{i+1}+\text { H.c. }\right)+\frac{1}{2} \sum_{i \neq j} V_{d d}(|i-j|) n_{i} n_{j},
$$

where $c_{i}^{\dagger}\left(c_{i}\right)$ is the creation (annihilation) operator of the fermion, $J=\sqrt{E_{R} V_{0}} \exp \left(-\pi^{2} \sqrt{V_{0} / E_{R}} / 4\right)$ with $E_{R}=$ $\hbar^{2} k^{2} / 2 m$ the recoil energy of the system, $V_{d d}(|i-j|)=$ $U_{d d} \iint \mathrm{d} x \mathrm{~d} x^{\prime} \widetilde{V}_{d d}\left(\left|x-x^{\prime}\right| / l_{\perp}\right) \omega^{*}\left(x-x_{i}\right) \omega^{*}\left(x^{\prime}-x_{j}\right) \omega(x-$ $\left.x_{i}\right) \omega\left(x^{\prime}-x_{j}\right)$ is the DDI between the dipoles at the positions of $x_{i}$ and $x_{j}$.

After the numerical calculation by setting $E_{R}, \hbar$, and $m$ as units, $\omega_{\perp} / \omega_{x}=\alpha$ and $V_{0} / E_{R}=\beta$, the $V_{d d}(|i-j|)$ is found to decay as $1 /|i-j|^{3}$ according to Fig 1. In Fig. 1, we show the Log-Log plot of $V_{d d} / v_{d d}(\theta)$ versus distances between dipoles in 1D optical lattices for different $\alpha$ and $\beta$ with $v_{d d}(\theta)=-C_{d d}[1+3 \cos (2 \theta)]$, where $\theta$ is the angle between the dipole direction and $x$ axis. When $\alpha$ is fixed

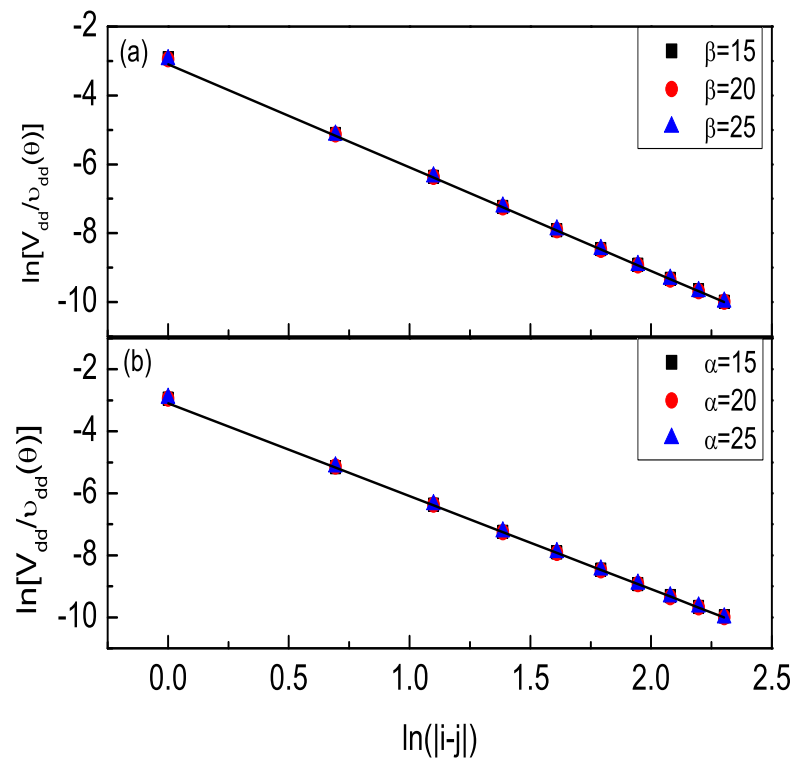

FIG. 1: (Color online) Log-Log plot of $V_{d d} / v_{d d}(\theta)$ versus distances between two dipoles in optical lattices for different $\alpha$ and $\beta$ with $v_{d d}(\theta)=-C_{d d}[1+3 \cos (2 \theta)]$. (a) We set $\alpha=20$ for different $\beta$, and the slop of the solid line is -3 ; (b) $\beta=20$ is fixed for $\alpha=15,20,25$, also the gradient of the fitting line is -3 .

for different $\beta$ (see Fig,1(a)), the gradient of the fitting line is -3 . In the Fig 1 (b), the slops of the fitting line is the same as the one in the Fig[1(a) where $\beta$ is fixed and $\alpha$ is changing. Also we can see when $\alpha$ is large enough, $V_{d d}$ changes little as $\alpha$ increases, because all the dipoles have been confined along the axial direction and can be seen as a one dimensional system. So we can simplify the Hamiltonian (3) as

$$
H=-J \sum_{i}\left(c_{i}^{\dagger} c_{i+1}+\text { H.c. }\right)+\frac{1}{2} V \sum_{i \neq j} \frac{n_{i} n_{j}}{|i-j|^{3}},
$$

with $V$ the strength of the DDI. For comparison, we also consider the system with only short-range interactions described by the following hamiltonian

$$
H=-J \sum_{i}\left(c_{i}^{\dagger} c_{i+1}+\text { H.c. }\right)+\frac{1}{2} V \sum_{\langle i, j\rangle} n_{i} n_{j},
$$

where only the nearest-neighbor interaction is considered and $\langle i, j\rangle$ means summation over nearest neighbors. In the present work, we only consider the case with repulsive interaction $V>0$. For simplicity, we take $\theta=\pi / 2$.

\section{RESULTS}

We shall study the ground state properties for both systems described by Hamiltonians (4) and (5) by the ex- 


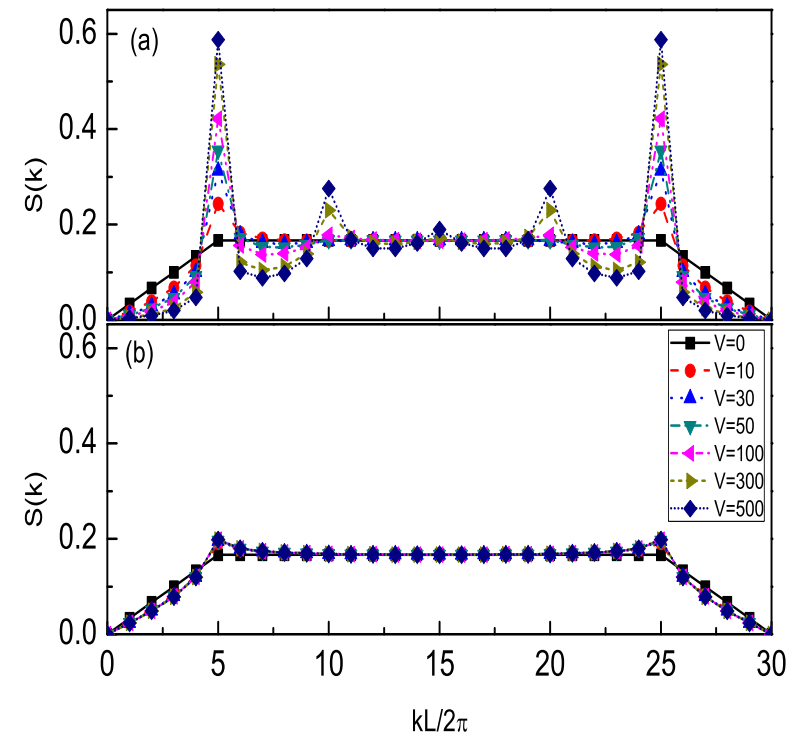

FIG. 2: (Color online) Static structure factors $S(k)$ for systems with $L=30, N=5$ and different $V$. (a) The dipolar Fermi system, and (b) the Fermi system with only the nearestneighbor interaction.

act diagonalization method. To give a concrete example, we focus on the system with five particles $(N=5)$ in a lattice with size $L=30$ under the periodic boundary condition. In order to gain some intuitive understanding of effect of long-range interactions, we first consider the limiting cases before presenting our calculated results. In the limit of $V / J \rightarrow 0$, the hopping term dominates and prevents the formation of crystal phase, and thus the difference of the long-range interaction and nearest-neighbor interaction is not obvious. However, in the strongly interacting limit of $V / J \rightarrow \infty$, the hopping term can be ignored, and the effect of long-range interaction becomes significant. The long-range repulsive interaction tends to repel particles to be equally spaced and form a solid like state. When apart from the strongly interacting limit, the hopping processes and quantum fluctuations prevent the formation of a perfect Wigner crystal.

To characterize the phase of Wigner crystal, we calculate the static structure factor, defined as

$$
S(k)=\frac{1}{L} \sum_{i, j} e^{i k(i-j)}\left[\left\langle n_{i} n_{j}\right\rangle-\left\langle n_{i}\right\rangle\left\langle n_{j}\right\rangle\right]
$$

where $k=2 m \pi / L$ with $m=0,1, \ldots, L$. The phase of Wigner crystal can be characterized by the onset of the $4 k_{F}$ peak in the static structure factor with $k_{F}=n \pi$ and $n=N / L$. When $V$ is small, we do not find obvious differences of $S(k)$ for systems with either long-range or short-range interaction. However, significant differences are emergent as the interaction strength increases

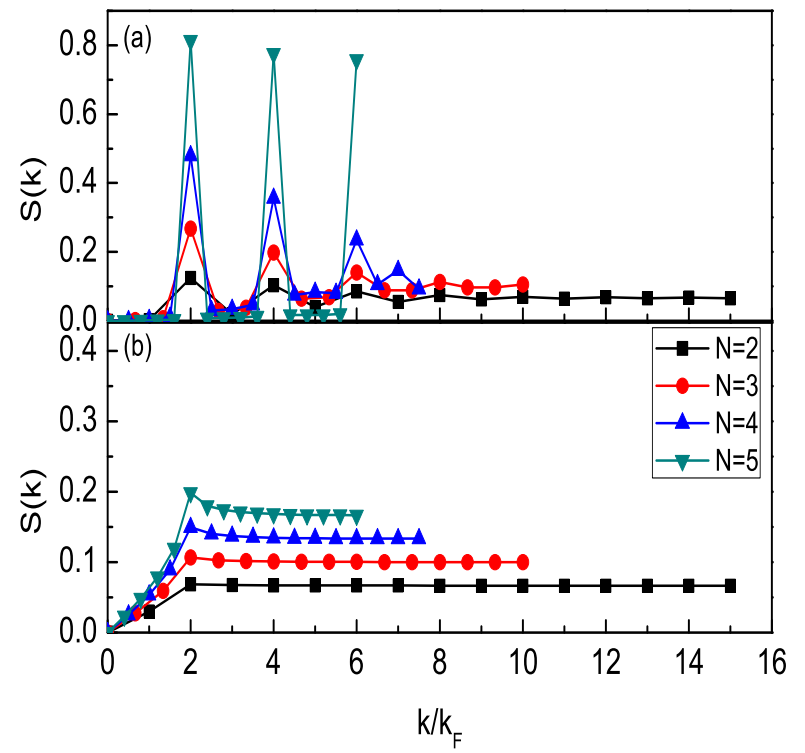

FIG. 3: (Color online) Static structure factors $S(k)$ vs $k / k_{F}$ for systems with $L=30, V=5000$ and different $N$. (a) is for the dipolar system, and (b) is for system with short range interaction.

to the strongly interacting limit. In Figure 2, we display the static structure factor $S(k)$ for different values of $V=0,10,50,100$ and 500 . For both systems, there is a peak at $k=2 k_{F}$ for $V=10$ despite that the peak for the system with long-range interaction is more obvious. As the interaction increases further, more peaks emerge at reciprocal lattice vectors $k L / 2 \pi N=$ integer for the system with the long-range interaction (Fig 22$)$. The height of peak increases with increasing $V$, evidencing the occurrence of the Wigner crystal state. While for the system with short range interaction described by Hamiltonian (5), from Fig 2(b) we can see that only peaks at $\pm 2 k_{F}$ occur and there are no other peaks appearing. Also, the height of peaks at $\pm 2 k_{F}$ increases more slowly with the increase of $V$ in contrast to the system with dipole-dipole interaction.

We also demonstrate data of static structure factor $S(k)$ versus $k / k_{F}$ in Fig 3 for systems with $L=30$, $V=5000$ and $N=2,3,4,5$. Systems with different $N$ display similar behaviors in the large $V$ limit. As shown in Fig.3(a), for systems described by Eq. (4) with different filling factors, peaks of $S(k)$ emerge at reciprocal lattice vectors $k / k_{F}=2 m$ with $m$ the integer. The appearance of peaks for various filling cases indicates that the Wigner crystal emerges when the filling is either commensurate or incommensurate with the optical lattice. For the systems described by Eq.(5), Fig.3(b) shows that there are no other peaks except at $k / k_{F}=2$.

Next we calculate the momentum distribution, which 


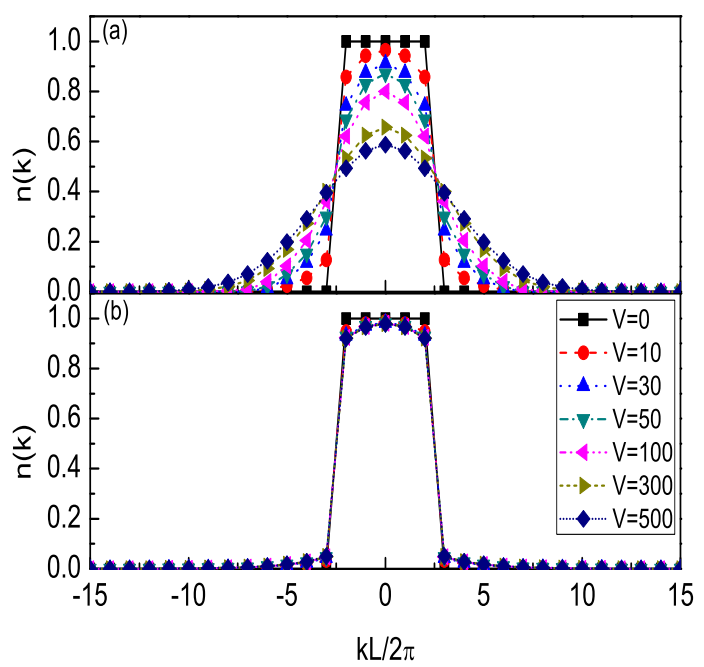

FIG. 4: (Color online) Momentum distributions for systems with $L=30, N=5$ and different $V$. (a) The dipolar Fermi system, (b) the Fermi system with nearest-neighbor interaction.

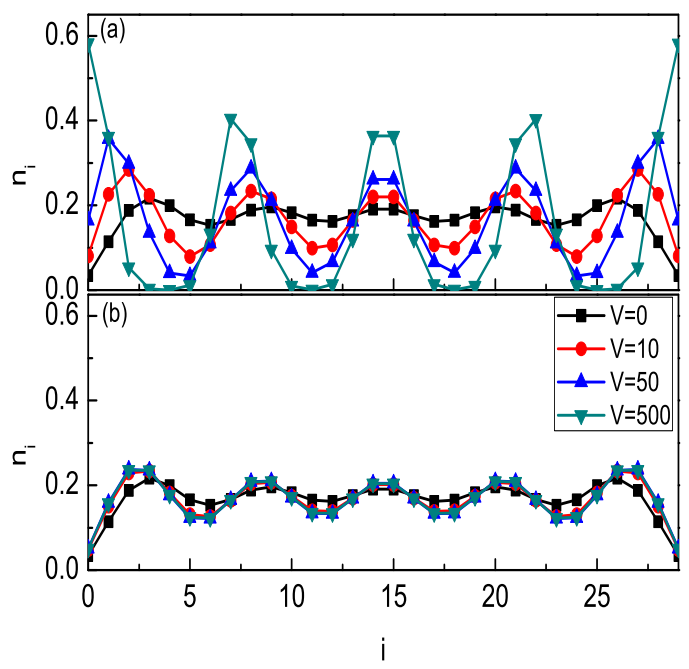

FIG. 5: (Color online) Density distributions for systems with $L=30, N=5$ and different $V$ under the open boundary condition. (a) The dipolar Fermi system, and (b) the Fermi system with nearest-neighbor interaction.

is defined by the Fourier transform with respect to $i-j$ of the one-particle density matrix with the form

$$
n(k)=\frac{1}{L} \sum_{i, j} e^{i k(i-j)}\left\langle c_{i}^{\dagger} c_{j}\right\rangle
$$

In Fig 4 we show momentum distributions for both sys- tems with different $V$. Fig 4 (a) is corresponding to the dipolar model, and Fig 4(b) is for the model with NN interactions. We also find the similarity of the two different models in the regime of small $V$. They show typical momentum step distribution of fermions. But with increasing $V$, the momentum densities of the dipolar model become fatter and lower (Fig 4(a)). At last for very strong $V$, the momentum distribution displays the Gaussian distribution which mainly comes from the Fourier transformation of the diagonal part of the density matrix, revealing that there is no overlap between the localized wave packets with the non-diagonal density matrix tending to vanish. In contrast to the case with DDI, the momentum distributions in Fig 4(b) change not obviously even for very strong $V$.

To gain an intuitive insight on the crystalline phase, we analyze the density distribution $n_{i}=\left\langle c_{i}^{\dagger} c_{i}\right\rangle$ for systems described by Hamiltonian (4) and (5) with open boundary conditions. In Fig. 5 we show density distributions of five particles for different $V$. As shown in Fig 5 (a) for the DDI systems, with increasing the long-range interaction, particles move apart each other with more sharp peaks emerging in the density profile. When $V$ is large enough, the density resembles five well-separated localized wave packs, which are totally apart from each other with the equilibrium positions of wave packets minimizing the interaction energy, characterizing the system evolved into the Wigner crystal regime 22]. As a comparison, the density distributions shown in Fig 5 (b) only change marginally with increasing the nearest-neighbor repulsion. No crystalline signal is detected even in the limit of strong repulsion.

Before ending this paper, we would like to discuss the validity of the approximation of the lowest transverse state in deriving the effective 1D Hamiltonian (2). Except of the requirement of $\omega_{\perp} / \omega_{x} \gg 1$, one also needs that the interacting energy $E_{D D I}$ induced by the dipolar interaction is much smaller than the energy of transverse confinement $\hbar \omega_{\perp}$. Different from the case with contact interaction, the interacting energy of the dipolar system is proportional to the interaction strength $V$, and thus the approximation is expected to break down if $E_{D D I}>\hbar \omega_{\perp}$. For the present case, the longitudinal kinetic energy is greatly suppressed due to the existence of the longitudinal optical lattice, i.e., $J \ll \hbar \omega_{x}$. Consequently, even when $V / J \gg 1$, the interacting energy is still much smaller than $\hbar \omega_{\perp}$ and thus the effective 1D description still holds true even in the regime of formation of Wigner crystal. Taking the case corresponding to Fig.2 as an example, if we take $\alpha=20$ and $\beta=20$, $J / \hbar \omega_{x} \sim 10^{-6}$, and therefore even for $V / J=500$ the $E_{D D I}$ is still much smaller than $\hbar \omega_{\perp}$. For systems with even stronger interaction or with large atom numbers, one can also tune $\alpha \gg 1$ to fulfill the requirement of $E_{D D I} \ll \hbar \omega_{\perp}$. In the future work, it would be also interesting to study the crossover from $1 \mathrm{D}$ to high-dimensional system when the transverse confinement decreases, for which the approximation of the lowest transverse state 
does not work well and new phenomena may appear 32 .

\section{SUMMARY}

In summary, we have studied the dipolar fermionic system with dipole-dipole interactions trapped in 1D optical lattices by means of the exact diagonalization method. We have shown that the static structure factor, momentum distribution and density distribution provide clear evidences for the existence of Wigner crystal, as the interaction energy overcomes the energy scale of hopping energy. We also compare our results of the dipolar system to the model with only nearest neighbor interactions, which exhibits no signatures of Wigner crystal even in the strongly interacting limit. Our study unveils the important role of the long range interaction in the formation of the Wigner crystal.

\section{Acknowledgments}

We thank X. L. Gao and S. Yi for helpful discussions. This work was supported by the 973 projects of the Ministry of Science and Technology of China (2011CB921700), NSF of China under Grants No. 10821403, No. 11174360 and No. 10974234.
[1] T. Lahaye, T. Koch, B. Frölich, M. Fattori, J. Metz, A. Griesmaier, S. Giovanazzi, and T. Pfau, Nature 448, 672 (2007).

[2] J. Stuhler, A. Griesmaier, T. Koch, M. Fattori, T. Pfau, S. Giovanazzi, P. Pedri, and L. Santos, Phys. Rev. Lett 95, 150406 (2005).

[3] K.-K. Ni, S. Ospelkaus, M. H. G. de Miranda, A. Pe'er, B. Neyenhuis, J. J. Zirbel, S. Kotochigova, P. S. Julienne, D. S. Jin, and J. Ye, Science 322, 231 (2008).

[4] S. Ospelkaus, A. Pe'er, K.-K. Ni, J. J. Zirbel, B. Neyenhuis, S. Kotochigova, P. S. Julienne, J. Ye, and D. S. Jin, Nat. Phys. 4, 622 (2008).

[5] D. Wang, B. Neyenhuis, M. H. G. de Miranda, K.-K. Ni, S. Ospelkaus, D. S. Jin, and J. Ye, Phys. Rev A 81, 061404 (2010).

[6] S. Yi and L. You, Phys. Rev. A 61, 041604(R) (2000); Phys. Rev. A 63, 053607 (2001).

[7] L. Santos, G.V. Shlyapnikov, P. Zoller, and M. Lewenstein, Phys. Rev. Lett. 85, 1791 (2000).

[8] S. Müller, J. Billy, E. A. L. Henn, H. Kadau, A. Griesmaier, M. Jona-Lasinio, L. Santos, and T. Pfau, Phys. Rev. A 84, 053601 (2011).

[9] Y. Kawaguchi, H. Saito, and M. Ueda, Phys. Rev. Lett. 96, 080405 (2006).

[10] L. Santos and T. Pfau, Phys. Rev. Lett. 96, 190494 (2006).

[11] J.-N. Zhang, L. He, H. Pu, C.-P. Sun, and S. Yi, Phys. Rev. A 79, 033615 (2009).

[12] K. Góral, L. Santos, and M. Lewenstein, Phys. Rev. Lett. 88, 170406 (2002).

[13] C. Trefzger, C. Menotti, and M. Lewenstein, Phys. Rev. Lett. 103, 035304 (2009).

[14] B. Capogrosso-Sansone, C. Trefzger, M. Lewenstein, P. Zoller, and G. Pupillo, Phys. Rev. Lett. 104, 125301 (2010).
[15] A. E. Golomedov, G. E. Astrakharchik, and Yu. E. Lozovik, Phys. Rev. A 84, 033615 (2011).

[16] S. Yi, T. Li, and C. P. Sun, Phys. Rev. Lett. 98, 260405 (2007).

[17] Z.-Y. Shi, R. Qi and H. Zhai, arXiv:1108.3510.

[18] R. -Z. Qiu, S.-P. Kou, Z.-X. Hu, X. Wan, and S. Yi, Phys. Rev. A 83, 063633 (2011).

[19] S. Sinha and L. Santos, Phys. Rev. Lett. 99, 140406 (2007).

[20] A. S. Arkhipov, G. E. Astrakharchik, A. V. Belikov, and Y. E. Lozovik, JETP Lett. 82, 39 (2005).

[21] R. Citro E. Orignac, S. De Palo, and M. L. Chiofalo, Phys. Rev. A 75, 051602(R) (2007).

[22] F. Deurezbacher, J. C. Cremon, and S. M. Reimann, Phys. Rev. A 81, 063616 (2010).

[23] S. Zöllner, G. M. Bruun, C. J. Pethick, and S. M. Reimann, Phys. Rev. Lett. 107, 035301 (2011).

[24] E. Wigner, Phys. Rev. 46, 1002 (1934).

[25] H. J. Schulz Phys. Rev. Lett. 71, 1864 (1993).

[26] H. Inoue and K. Nomura, J. Phys. A: Math. Gen. 39, 2161 (2006).

[27] Y. Tsukamoto and N. Kawakami, J. Phys. Soc. Jpn. 69, 149 (2000).

[28] M. Dalmonte, G. Pupillo, and P. Zoller, Phys. Rev. Lett. 105, 140401 (2010).

[29] G. E. Astrakharchik and M.D. Girardeau, Phys. Rev. B 83, 153303 (2011).

[30] R. M. Lee and N. D. Drummond, Phys. Rev. B 83, 245114 (2011).

[31] D. Jaksch, C. Bruder, J. I. Cirac, C. W. Gardiner, and P. Zoller, Phys. Rev. Lett. 81, 3108 (1998).

[32] See, for example, S. Komineas and N. R. Cooper, Phys. Rev. A 75, 023623 (2007). 